Rev. Adm. Saúde Vol. 17, № 69, Out. - Dez. 2017

http://dx.doi.org/10.23973/ras.69.58

RELATO DE CASO

\title{
Implantação de um modelo de descentralização de auditoria de contas hospitalares em um hospital de grande porte na região sul do Brasil
}

Implantation of a decentralization model for auditing hospital accounts in a large hospital in the southern region of Brazil

\section{Simone Brum da Silva ${ }^{1}$, Nathalia Santos Gonçalves ${ }^{2}$, Daniela Copetti Santos $^{3}$}

1. Enfermeira. Auditora em saúde no Hospital Ernesto Dornelles

2. Médica pelo Instituto Meridional, Passo Fundo - RS

3. Graduada em ciências biológicas. Professora efetiva do Instituto Federal de Educação, Ciência e Tecnologia, Farroupilha - RS

\section{RESUMO}

A auditoria é uma ferramenta de grande importância no controle e gestão de processos em instituições de saúde. Objetiva avaliar a qualidade dos processos de trabalho da assistência do serviço e os custos decorrentes da prestação da mesma. Ela colabora não só com a instituição, mas com os clientes, fornecedores e as próprias equipes de trabalho que desenvolvem esse serviço especializado, incluindo os profissionais da saúde, entre eles os enfermeiros. Na prática a auditoria atua de forma centralizada, desconectada com a origem dos registros, distante dos atores que produzem as ações, sejam elas de ordem financeira ou da qualidade da assistência. Análises feitas por uma equipe multidisciplinar visualizando as dificuldades encontradas em uma instituição hospitalar de grande porte na região de Porto Alegre, RS, tanto na auditoria assistencial, como na gestão de custos, motivaram a criar um modelo descentralizado de auditoria em saúde. Através desse modelo foi implementado extensões da auditoria nos setores ordenadores de despesas, possibilitando as revisões dos prontuários in loco, na origem, quebrando paradigmas culturais e financeiros da instituição, como revisões nos processos de trabalho e troca de saberes com as equipes, estreitando laços entre os 
setores de auditoria e assistência ao paciente, apresentando uma conta hospitalar muito mais transparente e fidedigna. Esse modelo além de ser uma atividade inovadora e única no Brasil, contribuiu para a diminuição das glosas, tornando-se uma ferramenta fundamental e indispensável para mensurar a qualidade do processo de trabalho da assistência de enfermagem e seu impacto econômico na instituição.

Palavras-chave: auditoria em saúde, instituição hospitalar, descentralização do sistema de saúde.

\section{ABSTRACT}

Audit is a tool of great importance in the control and management of processes in health institutions. It aims to evaluate the quality of the work processes of the service assistance and the costs of providing it. It collaborates not only with the institution, but with the clients, suppliers and the work teams themselves that develop this specialized service, including health professionals, among them nurses. In practice, the audit acts in a centralized way, disconnected with the origin of the records, away from the actors who produce the actions, be they financial or the quality of care. Analyzes made by a multidisciplinary team visualizing the difficulties found in a large hospital institution in the region of Porto Alegre, RS, both in the health care audit and in cost management, motivated the creation of a decentralized model of health audit. Through this model, auditing extensions were implemented in the expense computer sectors, enabling the on-site review of the records, in the origin, breaking the institution's cultural and financial paradigms, such as revisions in work processes and knowledge exchange with teams, strengthening ties between the audit and patient care sectors, presenting a much more transparent and trustworthy hospital account. This model, besides being an innovative and unique activity in Brazil, contributed to the reduction of glosses, becoming a fundamental and indispensable tool to measure the quality of the nursing care work process and its economic impact on the institution.

Keywords: health audit, hospital institution, decentralization of the health system.

\section{INTRODUÇÃO}

A auditoria longe de ser um instrumento punitivo recupera suas características de instrumento educativo, corretivo, continuo e de otimização. O auditor deve decidir sempre com respaldo técnico, científico, e com responsabilidade, não tem função fiscal e sim, orientador, pacificador, agente de mudança, de efetividade, de economicidade e eficiência, o auditor deve estar sempre atento aos registros, trabalhar com indicadores de qualidade e de assistência, proporcionando a viabilização de custos. Hoje, não somente a redução de 
custos deve estar em evidência, mas também, a qualidade do atendimento prestado, a qualidade do material adquirido e implementado.

$\mathrm{Na}$ atualidade, depara-se com um cenário no qual as instituições hospitalares passaram a ter um caráter organizacional com visão empresarial. Sendo assim, para garantir seus fins lucrativos, necessitam associar serviços de qualidade a baixo custo, garantindo a satisfação dos clientes. A auditoria é além de tudo, uma ferramenta de gestão para o diagnóstico de situações dentro do sistema de saúde, com vistas ao seu aperfeiçoamento.

Essa ferramenta tem sido usada pelos profissionais da saúde, em especial, os enfermeiros para avaliar a qualidade da assistência de enfermagem e os custos decorrentes da prestação desta atividade, além de fornecer subsídios para o planejamento e execução de atividades gerenciais, como orientação e capacitação da equipe de enfermagem para execução dos registros de enfermagem ${ }^{1}$.

Segundo pesquisadores ${ }^{2}$, a finalidade da auditoria de enfermagem está restrita à comprovação de pagamento de contas hospitalares, revendo glosas, realizando negociações entre representantes do hospital e do convênio. Além disso, eles já previam que em um futuro próximo, ela estaria associada à primeira finalidade, apontando inadequações da assistência de enfermagem, reformulando suas práticas, indicando processos de educação em serviço e delineando ações corretivas.

Segundo D`Innocenzo, et a $\beta$, cumpre salientar que a auditoria em enfermagem visa o controle de custos, a qualidade do atendimento ao cliente, o pagamento justo da conta hospitalar e a transparência das negociações, sempre embasada em uma conduta ética.

$\mathrm{Na}$ atualidade, para manterem-se no mercado competitivo, as instituições têm que aprender a associar baixos custos com excelência de qualidade para os seus clientes. Consequentemente, as instituições de cuidados de saúde têm sido compelidas a se organizarem como empresa, desenvolvendo visão de negócio para sobreviverem a estas mudanças no mercado ${ }^{4}$. A auditoria em enfermagem vem tomando novas dimensões ao longo dos anos e mostrando sua importância dentro das instituições hospitalares e operadoras de planos de saúde $^{5}$. Ela dever ser exercida e difundida nas instituições públicas e privadas, objetivando minimizar desperdício de materiais, medicamentos, equipamentos e recursos humanos ${ }^{6}$. No entanto, apesar de ser utilizada principalmente para fins contábeis, traduz-se em benefício não só para a instituição de saúde, uma vez que atinge tanto os pacientes e clientes, como para a própria equipe de enfermagem 6 .

Dun e Morgan7, afirmavam que a auditoria é um instrumento de administração utilizado na avaliação da qualidade do cuidado, baseado na comparação entre a assistência prestada e os padrões de assistência considerados aceitáveis.

Administrar qualidade refere-se, então, ao esforço para oferecer um bom produto ou serviço àqueles que se quer atingir. No caso de um serviço de saúde, o ideal seria oferecer um tipo de assistência que tenha o efeito 
desejado, de acordo com as expectativas dos usuários, dos técnicos prestadores de assistência, dos gestores dos serviços, dos financiadores e até dos fornecedores de materiais e equipamentos, entre outros atores privilegiados ${ }^{3}$.

A auditoria em enfermagem, na atualidade, é exercida e difundida nas instituições públicas e privadas, objetivando minimizar desperdício de materiais, medicamentos, equipamentos e recursos humanos ${ }^{6}$. No entanto, apesar de ser utilizada principalmente para fins contábeis, traduz-se em benefício não só para a instituição de saúde, uma vez que atinge tanto os pacientes e clientes, como para a própria equipe de enfermagem ${ }^{6}$.

Essa afirmação também é feita por Lopes et al. ${ }^{8}$ e Abdon et al. ${ }^{9}$, os mesmos dizem que com a aplicação da auditoria no processo da assistência, os benefícios atingem tanto pacientes como também a equipe e a instituição, com pacientes beneficiados com uma assistência de melhor qualidade e serviços oferecidos com eficácia.

\section{A implantação do sistema descentralizado de auditoria em saúde}

A partir de observações e estudos realizados por outros autores verifica-se que é de fundamental importância à criação de um modelo descentralizado de auditoria de contas hospitalares, o qual foi criado para ser utilizado em uma instituição hospitalar de grande porte na região de Porto Alegre, $\mathrm{RS}$, sendo assim relatado nesse trabalho. A criação desse modelo nasce com o objetivo de avaliar a necessidade de melhorar os processos dessa instituição tanto no âmbito assistencial, quanto na gestão de custos, buscando na gestão de contas dentro da auditoria eco para os seus maiores objetivos. Esse modelo surge no momento em que dificuldades são visualizadas e vivenciadas dentro dessa instituição hospitalar, tanto relacionadas às questões financeiras quanto a sua sustentabilidade.

Uma das maiores duvidas que surgiram durante a criação desse novo modelo é qual seria a contribuição que ele traria para a instituição, principalmente aqueles ligados a gestão de contas hospitalares. Entende-se que a instituição em seu modelo atual de auditoria não apresentava bons resultados, não havia um acompanhamento adequado ao tempo de faturamento da conta e a qualidade do que se apresentavam as operadoras.

Diante desse cenário apresentado até o presente momento foi proposto esse modelo aonde à instituição através de uma equipe multidisciplinar constituída por um gestor de contas, uma enfermeira auditora e uma farmacêutica analista de contas hospitalares, preocuparam-se com a reestruturação dos fluxos das contas hospitalar, abrangendo assim tanto a redução de custos, como a qualidade dos processos da assistência ao paciente. Esse modelo foi desenvolvido gradativamente, uma vez que a autora do projeto trabalha na instituição e participou ativamente dessa construção, desde a sua elaboração até a sua implantação. 
Durante o longo período que a auditoria percorreu no mundo, e pelas várias áreas que vem comprovando sua necessidade nas instituições, fica cada vez mais necessário e evidente que a auditoria seja encarada como uma importante aliada dos gestores em saúde, principalmente, pela contribuição efetiva de apoio e auxílio em medir e instruir as boas práticas dos serviços de qualidade empregadas nas instituições, contribuindo também significativamente, nas melhorias econômicas, criando um clima educativo e autossustentável nas instituições.

Atualmente, muito se fala em Instituições autossustentáveis na área da saúde, principalmente quanto a grande escassez de recursos públicos e privados para mantê-las, a auditoria, entra nesse contexto, como grande apoiadora da otimização desses recursos. A contribuição da auditoria na gestão promove a melhoria na integração das diferentes áreas ou setores, busca interface com os processos de trabalho já instituídos, podendo melhorar a comunicação interna dos setores, corrigindo falhas e reduzindo o desperdício dos recursos empregados.

Diante do exposto, a auditoria relacionada tanto na área pública como na privada, vêm em constante evolução, desde o seu surgimento até os dias atuais. Esse crescimento dá-se principalmente, pela nova visão de mercado da saúde, em relação às necessidades, tanto no âmbito das empresas prestadores de serviços como das empresas contratantes. Ambas, buscam melhorias dos serviços prestados ao cliente final, bem como, a qualidade das contas hospitalares apresentadas as operadoras de saúde, reunindo assim qualidade com custo econômico viável a essas empresas.

As instituições de saúde, por sua vez, buscando a excelência dos seus processos, vêm se apoiando de forma emergente nesse setor, com a crise financeira estabelecida, as empresas buscam a autossustentabilidade, e precisam lidar com clientes cada vez mais exigentes, nesse cenário a presença efetiva da auditoria nas instituições torna-se fundamental.

Dentro do contexto que envolve as instituições privadas de saúde, no cenário atual do ambiente organizacional, que está cada vez mais necessitando a adoção de medidas, técnicas, acompanhamento e controle que visam minimizar falhas e evitar desperdícios, a auditoria é uma grande aliada, pois busca ser um instrumento educativo, atuante em face às inúmeras dificuldades do sistema de saúde, portanto, deve ser considerada como um dos alicerces da manutenção da saúde da instituição.

A autora do artigo ainda relata que as suas atividades iniciais foram como enfermeira assistencial na própria instituição, atividades essas que a possibilitou adquirir os conhecimentos necessários às boas práticas da qualidade assistencial bem como, a gestão de recursos financeiros aplicados à referida área". Tendo em vista a necessidade da instituição de maior reforço no setor de auditoria de contas hospitalares, adentrei os processos de auditoria de enfermagem como auditora de contas hospitalares.

O trabalho desenvolvido na auditoria interna centralizada consistia na análise criteriosa dos prontuários dos pacientes internados na instituição, com objetivo 
principal de buscar ajustes na conta hospitalar apresentadas as operadoras de saúde, refletindo nas questões financeiras.

Com a percepção de que os erros categorizados eram quase sempre os mesmos, e que estes geravam as glosas nas contas hospitalares, iniciamos outro desafio, a busca da correção das falhas in loco, ou seja, na sua origem, na área assistencial. Esse modelo de descentralização da auditoria, até então, onde é sabido, é inédito no Brasil.

A descentralização da auditoria baseia-se na estruturação de equipes realocadas, de técnicos de enfermagem para técnicos de conta hospitalar, cargo específico criado com a finalidade de aproveitar a expertise e a técnica do colaborador. Estes trabalhando in loco, agindo como extensões da auditoria nos setores da origem do gasto, ligados a líderes auditores, responsáveis por cada área específica dentro da instituição, o número de técnicos pode variar de acordo com a demanda de cada área auditada e sua respectiva produção.

A auditora por hierarquia está subordinada ao gestor de contas que por sua vez está ligado ao gerente financeiro da instituição.

O técnico de conta hospitalar realiza o trabalho de revisão no prontuário médico e nas anotações de enfermagem, que consiste num conjunto de documentos padronizados e ordenados, destinados ao registro dos cuidados profissionais prestados pelos serviços de saúde. O objetivo principal é analisar os documentos e verificar a compatibilidade da cobrança realizada e se 0 processo de trabalho está sendo eficaz.

A cobrança ou a auditoria dos itens que foram auditados e analisados através de um software de gestão de saúde, chamado Tasy, A solução Philips Tasy para a gestão de prestadores de serviços em saúde é uma plataforma única, totalmente integrada e flexível, podendo ser configurada de acordo com as características e particularidades de cada realidade de negócio, onde apresenta todos os débitos da conta dispensados para determinado paciente. Caso o técnico em conta não encontre a evidência em forma de registro nos documentos, do uso do medicamento ou material, ele fará as devidas correções juntamente com a área de origem do gasto, a assistencial e ou médica. Essa conduta também influencia diretamente na educação dos envolvidos e principalmente no resultado final, como a glosa hospitalar.

Em busca de casos que comparem o estudo, verificou-se que há vários exemplos de instituições que utilizam o Tasy como ferramenta de gestão, uma delas está localizada na cidade de Fortaleza, no Ceará, o Hospital São Carlos a qual busca garantir a qualidade da assistência prestada com foco no cliente. Visando sempre a melhoria de seus serviços adotando assim um modelo de gestão empresarial envolvendo assim todos os seus colaboradores.

Com este foco, a instituição necessitava de um sistema de gerenciamento completo e passou a estudar profundamente várias opções do mercado que poderiam contemplar às suas necessidades reais. "Nossa opção pelo Tasy foi por ser um sistema de gerenciamento completo e integrado, parametrizável que contempla, dentre outras, a área assistencial, faturamento, financeiro e 
contábil; gera relatórios relevantes para tomadas de decisões estratégicas do hospital; promove segurança nas informações e facilita a auditoria nas transações", relata Marcia Real Machado, Administradora.

Contudo, viu-se, que o hospital mencionado, revisou os processos e só assim buscou as melhores práticas e mudanças necessárias. Viu-se também, que após tudo isso, os médicos passaram a usar prescrições eletrônicas que agilizam o processo e o deixam mais seguro na administração dos medicamentos.

João Neci Martins de Oliveira, gerente de projetos em TI do hospital São Carlos do Rio de Janeiro, RJ, relata:

"Durante estes 5 anos de utilização do Tasy tivemos grandes resultados, como a melhoria na integração entre os setores do hospital, assim como na disponibilidade e acesso a informação, evitando o retrabalho com digitações e diminuindo o custo com impressões. Antes da adoção do Tasy, trabalhávamos ilhados, tínhamos um retrabalho enorme com digitação de contas e lançamento de notas fiscais. Obtivemos grandes resultados também com a reestruturação de processos utilizados pelo hospital e modernização do nosso parque tecnológico. O mercado de saúde passa por uma fase de grandes mudanças e estar preparado para atender as necessidades tecnológicas desse mercado é fundamental para o nosso sucesso".

O Hospital Vicente de Paulo (HVSP) é um hospital geral com alta tecnologia que desde 2010 começou a implantar o sistema Philips Tasy em suas operações. Desde então, o Hospital que figura na lista dos 40 melhores hospitais e clínicas da América Latina, começou a apresentar aumento no faturamento: em 2012 o Hospital faturou 19,37\% a mais em relação ao ano de 2011 e $13,76 \%$ nos pagamentos de convênios.

Já Rafael Ramos de Souza, analista de custos e orçamento destaca:

"Na área financeira com a implantação do Tasy, o hospital obteve ganhos tanto na lucratividade, quanto na veracidade das informações. "No encerramento do exercício de 2010, quando se iniciou a implantação do sistema Tasy, o HSVP tinha um faturamento médio de $\mathrm{R} \$ 8,7$ milhões por mês e um recebimento médio de $R \$ 8,5$ milhões mensais. As despesas operacionais estavam na média de $R \$ 9,5$ milhões mensais, 0 que gerava um déficit médio de $\mathrm{R} \$ 1$ milhão. Após a implantação dos módulos financeiros de faturamento e de atendimento, o HSVP passou a ter mais controle dos eventos gerados em cada uma dessas áreas".

Viu-se que estas instituições, antes de adquirirem o sistema Philips Tasy, a gestão apresentava fragilidades e vulnerabilidades para a sustentabilidade da organização, pois não havia o suporte de um sistema que auxiliasse na tomada de decisão através de indicadores de desempenho. 
E com a busca de melhoria foi fundamental a aquisição do processo Tasy, pois com isso, o Hospital São Vicente de Paulo vai melhorar seus processos e gerenciar cada vez melhor, todos os setores da instituição.

André Mallmann, gerente de TI do HSVP, complementa que o sistema é flexível e parametrizável, sendo totalmente aderente aos processos internos da instituição. Outra característica importante, segundo ele, é que o sistema é aberto em relatórios e indicadores, o que proporciona liberdade para a área de TI sustentar e criar os indicadores de acordo com a realidade da empresa. Ele ainda enfatiza a velocidade na resolução dos problemas com o suporte prestado pela Philips.

Vale ressaltar que, o técnico de conta hospitalar aproveita sua experiência da prática de técnico de enfermagem, para avaliar os gastos inerentes aos procedimentos auditados.

A auditora por sua vez, posiciona-se nesse cenário como líder dos grupos de técnicos em conta hospitalar, organizando, orientando, avaliando e coordenando as rotinas e fluxos da revisão dos registros, bem como a elaboração de treinamentos e reciclagem dos profissionais envolvidos. Diante dessa concepção, a percepção do auditor em relação aos métodos, finalidade e os parâmetros legais que sustentam na prática a sistematização do processo de trabalho, são fundamentais para um bom resultado.

Dessa forma, foi incorporada na rotina da autora desse artigo e da equipe multidisciplinar que atuavam junto a ela as análises de dados, como tempo e movimento das etapas do processo de auditoria da conta do paciente. Resultando em ações para diminuição do tempo passivo que a conta sofre em função das inconformidades.

Para discorrer sobre a conta hospitalar e o tempo passivo da mesma, é preciso entender que o setor de faturamento da instituição é o responsável pela última revisão geral da conta e envio de relatório de cobrança as operadoras, respeitando os prazos de entrega contratuais pré-estabelecidos. É o resultado de todos os gastos auditados pelo técnico de conta hospitalar, que podem ser em atendimento ambulatorial ou internado.

O setor de faturamento da operadora avalia a cobrança e faz o pagamento aos serviços prestados pela instituição, sempre que houver consenso da cobrança, caso discorde, realiza a glosa da conta ou de itens que apresentam inconformidades com as cláusulas contratuais ou falta de evidências de registros do serviço prestado. O tempo de faturamento da conta do paciente é medido, portanto, através das etapas que ela passa, o fluxo foi construído a medida que as necessidades de adequação surgiam, acertos e mudanças foram realizados com estratégias específicas que permitiram que a conta permanecesse o menor tempo possível em cada etapa. Passamos a medir através da auditoria a ocorrência dos erros recorrentes nos documentos, registros médicos e de enfermagem, dos pacientes, avaliando as melhores medidas preventivas para reduzir ao máximo a reincidência. 
Ao final de dois anos de análise, validando o processo, chegamos as seguintes conclusões: a qualidade da conta apresentada no que se refere em apresentação, clareza, transparência nas evidências de gastos, fidedignidade das informações, quebra de paradigmas culturais e o resgate de laços intersetoriais, foram superiores as nossas expectativas. Quanto a apresentação da conta em relação à redução de perdas financeiras, foram significativamente superiores ao proposto inicialmente pelo projeto, hoje validado como modelo institucional, levando a instituição a números nunca antes alcançados de faturamento. Tanto deu certo que está impulsionando outras instituições do setor hospitalar a conhecer na prática o modelo "Descentralizado da Auditoria de Contas Hospitalares".

Com todo este estudo aplicado na instituição, fez-se um trabalho árduo e gratificante, contando com a ajuda de outros profissionais e suas expertises para colocar o processo em prática, pois não se encontrou nenhum outro estudo teórico sobre a descentralização da auditoria.

\section{CONSIDERAÇÕES FINAIS}

Depois desses dois anos de implantação do novo modelo de auditoria ainda temos muito a percorrer, melhorando dessa forma os gastos que ainda são gerados desnecessariamente na instituição e buscando atender aos nossos objetivos através de um serviço de grande excelência em auditoria hospitalar. Não esquecendo, é claro, dos grandes resultados que já foram apresentados após esse tempo.

Os resultados da descentralização da auditoria permitem viabilizar a validade da implantação desse sistema em outras instituições, visto que não existem relatos na literatura de outros espaços de saúde que tenham adotado esse modelo.

A auditoria de enfermagem, hoje, abrange uma vasta área de atuação. Enfermeiros auditores estão presentes nas instituições de saúde como: serviços de educação continuada, serviço de faturamento, serviços de credenciamento para realização de vistoria técnica da rede, autorizações pela verificação e análise da compatibilidade dos procedimentos solicitados com a realidade contratual entre operadora, prestador de serviços e cliente, serviços de contas médicas na orientação e coordenação dos auxiliares de revisão de contas e nos serviços de auditoria de enfermagem e médica propriamente dita, entre vários outros.

A auditoria de enfermagem, quando implantada nos serviços de saúde e operadoras, estará interligada com todas as outras áreas da empresa e essa comunicação entre setores possibilitará o sucesso deste trabalho.

A descentralização da auditoria, como modelo instituído, proporcionou nova postura corporativa, que atualmente impulsiona outras instituições que buscam a melhoria dos seus processos, uma vez que esse modelo é único no Brasil até onde sabemos. 


\section{REFERÊNCIAS}

1. Vieira APT. Enfermeira auditora uma ferramenta importante para a qualidade do serviço em saúde em hospitais privados. - Revista Especialize On-line IPOG- 8ª Edição - Goiânia - 2014; 9(01): 1-13.

2. Scarparo AF, Ferraz CA. Auditoria em Enfermagem: identificando sua concepção e métodos. Rev Bras Enferm, Brasília 2008; 61(3): 302-5. http://dx.doi.org/10.1590/S0034-71672008000300004

3. D'innocenzo M, Feldman LB, Fazenda NRR, Helito RAB, Ruthes RM. Indicadores, auditoria e certificações: ferramentas de qualidade para gestão em saúde. São Paulo: Martinari, 2006. 206 p.

4. Bichanga CA. Gerenciamento de custos através de avaliações de cobranças e sua efetividade. In: 7ํㅡㄹ ENFTEC; 2000; São Paulo. Anais eletrônicos. São Paulo, 2000.

5. Motta ALC. Auditoria de Enfermagem no Processo de Credenciamento. São Paulo: látria, 2003. 152 p.

6. Silva MVS, Silva LMS, Dourado HHM, Nascimento AAM. Limites e possibilidades da auditoria em enfermagem e seus aspectos teóricos e práticos. Rev Bras Enferm, Brasília 2012 mai-jun; 65(3): 535-8.

http://dx.doi.org/10.1590/S0034-71672012000300021

7. Dunn HW. \& Morgan EM. - The nursing audit. New York, National League for Nursing, 1968. 38 p.

8. Lopes MMB, Carvalho JN, Backes MTS, Erdmann AL, Meirelles BHS.

Políticas e tecnologias de gestão em serviços de saúde e de enfermagem. Acta Paul Enferm; 2009; 22(6): 819-27.

9. Abdon JB, Dodt RCM, Vieira DP, Martinho NJ, Carneiro EP, Ximenes LP. Auditoria dos registros na consulta de enfermagem acompanhando o crescimento e desenvolvimento infantil. Rev Rene; 2009; 10(3):90-6.

Recebido: 3 outubro 2017. Publicado: 16 outubro 2017

Correspondência: Simone Brum da Silva. E-mail: simone.brum@hed.com.br

Conflito de Interesses: os autores declararam não haver conflito de interesses. 
(C) This is an Open Access article distributed under the terms of the Creative Commons Attribution License, which permits unrestricted use, distribution, and reproduction in any medium, provided the original work is properly cited 